\title{
Adjoints of large simulation codes through Automatic Differentiation
}

\author{
Laurent Hascoët* - Benjamin Dauvergne* \\ * INRIA Sophia-Antipolis, équipe TROPICS \\ 2004 Route des lucioles, BP 93, 06901 Sophia-Antipolis, France \\ \{Laurent.Hascoet, Benjamin.Dauvergne\}@sophia.inria.fr
}

\begin{abstract}
Adjoint methods are the choice approach to obtain gradients of large simulation codes. Automatic Differentiation has already produced adjoint codes for several simulation codes, and research continues to apply it to even larger applications. We compare the approaches chosen by existing Automatic Differentiation tools to build adjoint algorithms. These approaches share similar problems related to data-flow and memory traffic. We present some current state-of-the-art answers to these problems, and show the results on some applications.

RÉSUMÉ. Les méthodes adjointes sont largement utilisées pour obtenir des gradients de simulations de grande taille. La Différentiation Automatique est une méthode de construction des codes adjoints qui a déjà été appliquée à plusieurs codes de taille réaliste, et les recherches visent des codes encore plus gros. Nous comparons les approches choisies par les principaux outils de Différentiation Automatique pour construire des codes adjoints, en mettant l'accent sur les problèmes de flot de données et de consommation mémoire. Nous présentons des développements récents dans l'application d'un principe classique de compromis stockage-recalcul, et nous montrons nos résultats expérimentaux préliminaires.

KEYWORDS: Automatic Differentiation, Reverse Mode, Checkpointing, Adjoint methods, Gradient

MOTS-CLÉS : Différentiation Automatique, Mode Inverse, Checkpointing, Etat Adjoint, Code Adjoint, Gradient
\end{abstract}

$1^{\text {re }}$ soumission à $R E M N$, le 05/02/07 


\section{Introduction}

Modern Scientific Computing increasingly relies on the computation of several sorts of derivatives. Obviously, derivatives play a natural role in the basic simulation activity, as well as in most of the mathematics that model the systems to simulate. But we are now witnessing a sharp increase in the use of derivatives, made possible by the impressive power of present computers on one hand, and probably by new programming concepts and tools, such as Automatic Differentiation (AD), on the other hand.

The present issue provides ample illustration of these novel uses of derivatives. Now that computing capacities technically allow for it, researchers explore new usages of derivatives. To quote some examples, simulation of a complex system in a neighborhood of some initial configuration is no longer limited to a simple linear approximation. Second- and higher-derivatives can provide a much more accurate simulation, and are now affordable. Similarly, the development of gradient-based optimization of complex systems requires efficient gradients through adjoints. Researchers are exploring the computation of these gradients even for very long and expensive instationnary simulations (Mani et al., 2007). Further, gradient-based optimization, which several years ago was restricting to approximate quasi-Newton methods, is now considering true Newton methods, which require second-order Hessian derivatives. Even further, the Halley method is being considered again, and it requires a third-order derivative tensor. Second-order derivative information is also the key to the sensitivity of the optimum itself, leading to so-called robust design.

In this small catalog, Adjoint Codes rank among the most promising kinds of derivatives, because gradients are crucial in Optimization, and because the adjoint method can return a gradient at a cost essentially independent from the number of input parameters. The justification for this will be sketched in Section 2. Applications in CFD or structural mechanics require gradients for sensitivity analysis and optimal shape design. Applications such as climate research, meteorology, or oceanography, require gradients for sensitivity analysis and inverse problems e.g. variational data assimilation. Their number of input parameters is often several millions, which makes it impossible to compute the gradient with direct approaches such as divided differences. The adjoint method is the appropriate strategy to build these gradients, and therefore adjoint codes have been written for several applications, often by hand at a huge development cost. Moreover, hand-written adjoint codes were often built from simplified models only, to reduce development cost, but this discrepancy produced annoying effects in the optimization loop. But the increasing complexity of e.g. simulations of turbulent flow by LES models makes this simplification even more hazardous. Present AD tools can automatically build efficient adjoint codes for these very large and complex simulations.

AD tools are still research tools. The applications shown in section 5 demonstrate that $\mathrm{AD}$ can now address simulations of a very decent size, and are making rapid progress in this respect. However they will maybe never become black-box tools like 
compilers. Interaction with the end-user is unavoidable to differentiate the largest codes.

In this article, we will introduce the principles of $\mathrm{AD}$ in Section 2, emphasizing the notions behind AD adjoint codes. We will present in Section 3 the existing AD tools that can produce adjoint codes and we will try to compare their specific strategies. Because optimization of instationnary simulations is one challenge of the years to come, we will study in Section 4 the specific problems that must be addressed in the case of very large and time-consuming codes. Section 5 will present some applications of our AD tool Tapenade to large instationnary simulation codes, with our first realistic measurements of the performance of AD-generated adjoint codes.

\section{Building Adjoint Algorithms through Automatic Differentiation}

Automatic Differentiation (AD) is a technique to evaluate derivatives of a function $F: X \in \mathbb{R}^{m} \mapsto Y \in \mathbb{R}^{n}$ defined by a computer program $\mathrm{P}$. In $\mathrm{AD}$, the original program is automatically transformed or extended to a new program $\mathrm{P}^{\prime}$ that computes the derivatives analytically. For reference, we recommend the monograph (Griewank, 2000), selected articles of recent conferences (Corliss et al., 2001; Bücker et al., 2006), or the AD community website www . autodiff .org.

After some formalization of Automatic Differentiation in general in section 2.1, we focus in section 2.2 on the structure of AD-generated adjoint codes, which we call adjoint algorithms. In section 2.3, we underline the principal difficulty that these adjoint algorithms must overcome.

\subsection{Principles of Automatic Differentiation}

The first principle of Automatic Differentiation is to consider any numerical program as a mathematical function, obtained by composing the elementary functions implemented by each simple statement. The analytic derivatives of the complete program can therefore be computed using the chain rule of calculus. Since these are analytic derivatives, they have the same level of accuracy as the given numerical program, and are free from the approximation error which is inherent to the "Divided Differences" $(F(X+\varepsilon)-F(X)) / \varepsilon$

The second principle of AD is that it is "Automatic", i.e. the end user doesn't need to actually write the derivative program. This task is performed by a tool or by an appropriate environment, so that producing the derivative code actually costs very little. This is especially important when the original code may be modified several times to embed new equations, new discretization choices or new algorithms. In most cases, actual differentiation of code can be called from a Makefile. The differentiated code is regarded as some intermediate step in the compile process, and ideally should never be modified or post-processed by hand. Unfortunately, in reality this still occurs 
sometimes, but it is considered a weakness and AD tools are striving to progressively eliminate these hand modification stages.

Let's now introduce a bit of formalization. Consider a numerical program $P$ that implements a function $F$. Among the outputs of $P$, suppose we identify a subset $Y$ of variables that we want to differentiate. Symmetrically, among the inputs of $P$, suppose we identify a subset $X$ of variables with respect to which we want to differentiate $Y$. Both $X$ and $Y$ are multi-dimensional in general, and must consist of variables of a continuous type e.g. floating-point numbers. The $X$ are often called the "independents" and $Y$ the "dependents". We are looking for the derivatives of $F$ at the current input point $X=X_{0}$, and we will assume that there exists a neighborhood around $X_{0}$ inside which the control flow of $\mathrm{P}$ remains the same. This means that all conditional branches, loops, array indices, or other address computation are the same for any input points in this neighborhood of $X_{0}$. This is apparently a strong assumption, since the flow of control of a program usually may change a lot when the inputs change, but in practice this assumption is reasonable and leads to useful and reliable derivatives. In this neighborhood, execution of $\mathrm{P}$ is equivalent to the execution of a (possibly very long) sequence of simple statements $I_{k, k=1 \rightarrow p}$ :

$$
\mathrm{P}=I_{1} ; I_{2} ; \ldots I_{p-1} ; I_{p} .
$$

Calling $f_{k}$ the mathematical function implemented by $I_{k}$, we know that the function $F$ computed by $\mathrm{P}$ is:

$$
F=f_{p} \circ f_{p-1} \circ \cdots \circ f_{1} .
$$

Calling $W_{k}$ the set of all intermediate values after statement $I_{k}$, defined by $W_{0}=X_{0}$ and $W_{k}=f_{k}\left(W_{k-1}\right)$, we can use the chain rule to compute the derivative of $F$ :

$$
F^{\prime}\left(X_{0}\right)=f_{p}^{\prime}\left(W_{p-1}\right) \cdot f_{p-1}^{\prime}\left(W_{p-2}\right) \ldots \ldots f_{1}^{\prime}\left(W_{0}\right)
$$

and this can be implemented right away by a new program $\mathrm{P}^{\prime}$, called the differentiated program. The goal of Automatic Differentiation is to produce such a program $\mathrm{P}^{\prime}$ automatically from $P$, for instance through program augmentation or program tran Sformation, or even as an additional phase during compilation of $\mathrm{P}$.

It turns out in practice that this full Jacobian matrix $F^{\prime}(X)$ is expensive to compute and for most applications is not really necessary. Instead, what applications often need is either the "tangent" derivative:

$$
\dot{Y}=F^{\prime}(X) \cdot \dot{X}=f_{p}^{\prime}\left(W_{p-1}\right) \cdot f_{p-1}^{\prime}\left(W_{p-2}\right) \ldots \ldots f_{1}^{\prime}\left(W_{0}\right) \cdot \dot{X}
$$

or the "adjoint" (or "reverse" or "cotangent") derivative:

$$
\bar{X}=F^{\prime t}(X) \cdot \bar{Y}=f_{1}^{\prime t}\left(W_{0}\right) \ldots \ldots f_{p-1}^{\prime t}\left(W_{p-2}\right) \cdot f_{p}^{\prime t}\left(W_{p-1}\right) \cdot \bar{Y}
$$

Intuitively, the tangent derivative $\dot{Y}$ is the directional derivative of $F$ along a given direction $\dot{X}$. The adjoint derivative $\bar{X}$ is the gradient, in the input space, of the dotproduct of $Y$ with a given weighting $\bar{Y}$. Both formulas for tangent and adjoint derivatives are better evaluated from right to left, because $\dot{X}$ and $\bar{Y}$ are vectors and the $f_{k}^{\prime}$ are 
matrices, and matrix $\times$ vector products are much cheaper than matrix $\times$ matrix. Moreover each $f_{k}^{\prime}$ is very sparse and simple. If $I_{k}$ is an assignment to a variable $\mathrm{v}_{k}$ of an expression that uses $d$ other variables, $f_{k}^{\prime}$ is basically an identity matrix in which the $k^{t h}$ row is replaced with a vector with only $d$ non-zero entries. Choosing this right to left evaluation order, one $\dot{Y}$ or one $\bar{X}$ costs only a small multiple of the cost of $F$ itself, independently of the sizes of $X$ and $Y$. The slowdown factor from $\mathrm{P}$ to $\mathrm{P}^{\prime}$ reflects the cost ratio between elementary operations and their derivative, which ranges between one and four. It depends on the actual code and on the optimizations performed by the compiler. In practice it is typically 2.5 with the tangent mode and 7 with the adjoint mode. The key observation is that this ratio is essentially independent from the length of the program $\mathrm{P}$ and from the dimensions of the independent input $X$ and dependent output $Y$. The reasons for the higher cost ratio of the adjoint mode will become apparent when we study this mode in more detail.

For most of the applications we are targeting at, the required derivative is actually a gradient of a scalar cost function. For optimization, the cost function can be a physical value such as the lift/drag ratio of an airplane. For inverse problems and optimal control, it will be the least-square discrepancy between the computed final state and a target state or more generally between all computed intermediate states and a target trajectory. In these cases, the dimension $m$ of $X$ is large whereas $Y$ is a single scalar. One run of the tangent code would return only one scalar $\dot{Y}$, and $m$ runs are needed to build the complete gradient. On the other hand, the adjoint algorithm returns this complete gradient in just one run. In the sequel of this article, we will therefore focus on the adjoint mode of AD.

\subsection{The adjoint mode of Automatic Differentiation}

The adjoint mode of AD builds a new code $\overline{\mathrm{P}}$ that evaluates

$$
\bar{X}=F^{\prime t}(X) \cdot \bar{Y}=f_{1}^{\prime t}\left(W_{0}\right) \ldots f_{p-1}^{\prime t}\left(W_{p-2}\right) \cdot f_{p}^{\prime t}\left(W_{p-1}\right) \cdot \bar{Y}
$$

from right to left. Therefore it computes $f_{p}^{\prime t}\left(W_{p-1}\right) \cdot \bar{Y}$ first, and this needs the values $W_{p-1}$ that the original program P knows only after statement $I_{p-1}$ is executed. This implies a specific structure for $\overline{\mathrm{P}}$ which is often surprising at first sight: it consists of an initial run of a copy of $\mathrm{P}$, followed by actual computation of the intermediate gradients $\bar{W}_{k, k=p \rightarrow 0}$ initialized with

$$
\bar{W}_{p}=\bar{Y}
$$

and computed progressively by

$$
\bar{W}_{k-1}=f_{k}^{\prime t}\left(W_{k-1}\right) \cdot \bar{W}_{k}
$$

for $k=p$ down to $1 . \bar{X}$ is found in the final $\bar{W}_{0}$.

Consider the following very simple example for $\mathrm{P}$, from inputs $\mathrm{a}$ and $\mathrm{b}$ to result $\mathrm{c}$ : 


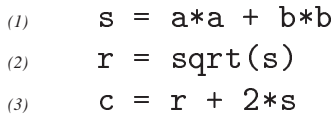

Essentially, $\overline{\mathrm{P}}$ will be the following, from inputs $\mathrm{a}, \mathrm{b}$, and $\overline{\mathrm{c}}$ to results $\overline{\mathrm{a}}$ and $\overline{\mathrm{b}}$

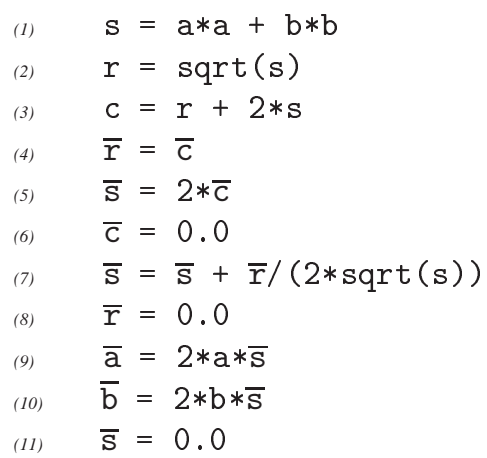

where lines $\overline{\mathrm{P}}:(1-3)$ are the copy from $\mathrm{P}$, usually called the forward sweep. They are followed by the backward sweep, which is made of the differentiated instructions for each original statement, in reverse order, namely $\overline{\mathrm{P}}:(4-6)$ from $\mathrm{P}:(3), \overline{\mathrm{P}}:(7-8)$ from $\mathrm{P}:(2)$, and $\overline{\mathrm{P}}:(9-11)$ from $\mathrm{P}:(1)$. The reader can easily check each differentiated instructions set by building the transposed Jacobian times vector product $f_{k}^{\prime t}\left(W_{k-1}\right) \cdot \bar{W}_{k}$ for $k=3$ down to 1 .

Let's look at the cost of computing the gradient $\overline{\mathrm{a}}$ and $\overline{\mathrm{b}}$ for this simple example, by counting the number of arithmetic operations. P itself costs 6 arithmetic operations. The naive divided differences approach would require at least three runs of $P$, i.e. a total cost of $3 * 6=18$ operations. Computing the same gradient using the tangent mode (not shown here) would require two executions of the tangent mode for a total cost of $15+15=30$. This cost can be easily reduced to $15+9=24$ by sharing the original values between the two derivatives computations. This is more expensive than divided differences. In general the two costs are comparable for large programs, but the accuracy of tangent derivatives is clearly better. Finally, $\overline{\mathrm{P}}$ computes the gradient in just one run, at a cost of 15 arithmetic operations, which is already better than the other approaches. This advantage becomes even higher as the number of input variable grows. The slowdown factor from $\mathrm{P}$ to $\overline{\mathrm{P}}$ is here 2.5 .

The structure of the adjoint algorithm $\overline{\mathrm{P}}$ becomes even more surprising when control comes into play. The control path which is actually taken in the program $\mathrm{P}$ and therefore in the forward sweep of $\bar{P}$ must be taken exactly in reverse in the backward sweep of $\bar{P}$. One way to do that is to remember all control decisions during the forward sweep, by storing them on a stack. During the backward sweep, the control decisions are read from the stack when needed, and they monitor the control of the backward sweep. With this approach, conditionals from $\mathrm{P}$ become conditionals in $\overline{\mathrm{P}}$, and loops in $\mathrm{P}$ become loops in $\overline{\mathrm{P}}$, although their iteration order is reversed. 


\subsection{The taping problem of adjoint $A D$}

However, there is a problem lying in the reverse order of derivative computations in adjoint $\mathrm{AD}$, which uses values from $\mathrm{P}$ in the reverse of their computation order. $\mathrm{Al}$ though we carefully designed the example in section 2.2 to avoid this, real programs often overwrite or erase variables. Overwriting actually c annot be avoided in real simulation programs, which are iterative in essence. For adjoint AD, an erased variable must be restored if the erased value is used in a derivative computation. This has a cost, whichever strategy is used.

If a value is needed which is not available, because it has been erased, there are basically three tactics to make it available again:

- The desired value may have been stored in memory just before erasal. This is the fundamental tactic and it motivates the name we give to this "taping" problem.

- The desired value may be recomputed forward by repeating the statement that defined it, provided the inputs to this statement are themselves available.

- In a few situations, the desired value may be deduced from a statement that uses it, provided that the statement is "invertible" and that the other inputs to this statement and its output are available. For example if a and c are available, one can invert statement $\mathrm{a}=\mathrm{b}+\mathrm{c}$ to make $\mathrm{b}$ available.

Adjoint $\mathrm{AD}$ on large programs must use a clever combination of these three tactics to compute the gradient efficiently. We will see that, whatever the strategy, it will always include some amount of storage.

The number of values that are overwritten by P grows linearly with the execution time of $P$. Thus this problem becomes even more crucial for the very long codes that we are now considering, such as instationnary simulations.

\section{Automatic Differentiation approaches and tools: Advantages and Drawbacks}

There exist today a large number of AD tools. In this section we will select only a subset of them, which seem to us the most active tools, and which are representative of the existing different approaches. We aim at being objective here, although we are developing one of these tools, Tapenade. One can find a more complete catalog on the $\mathrm{AD}$ community website www . autodiff . org. We will emphasize how the approaches behave for building adjoints of large simulation codes. We will first present the general picture in section 3.1, together with a summary in table 1 . We then compare the merits of the main alternatives, namely Overloading $v s$ Program Transformation in section 3.2 and strategies for restoring overwritten values in section 3.3. 


\subsection{AD approaches and tools}

Traditionally, the first distinction among AD tools is code overloading vs explicit program transformation. Since program transformation tools are harder to implement, code overloading AD tools came earlier. The principal member of the overloading class is the AD tool Adol-C (Griewank et al., 1996). Adol-C applies to C++ codes, using the overloading capacities of Object-Oriented style. Similarly for MATLAB, available AD packages such as ADMAT (Verma, 1999), ADiMat (Bischof et al., 2003), and recently MAD (Forth, 2006), rely on overloading. ADiMat mixes overloading with some amount of code transformation. All overloading tools offer an implementation of the tangent mode, and can often compute other tangent directional higher-order derivatives such as Taylor developments.

Some of the overloading tools also offer adjoint AD capacities, but at a cost that we will discuss in section 3.2. To our knowledge, overloading-based tools have produced adjoint algorithms only for relatively small applications. For adjoints of large simulation codes, overloading becomes too expensive and program transformation tools are compulsory. These tools share common organization principles, that we can summarize as four successive phases:

1) Parse the complete given program, with all subroutines and include files, and build an internal representation.

2) Perform a number of global static analyses on this internal representation. Most of these are data-flow analyses that help produce a better result. Some of these analyses are completely specific to differentiation.

3) Build the differentiated program, in the internal representation format.

4) Regenerate a differentiated source code.

Phases 1 and 2 obviously look very much like what is done in compilers. The internal representation makes the tool less dependent on the target language. This idea was present right from the design stage for Tapenade (Hascoët et al., 2004) and OpenAD (Utke et al., 2006), for which phases 2 and 3 are language-independent. This makes extensions to new languages easier. There is a potential extra level of flexibility with OpenAD, which publishes an API allowing a programmer to define new code analyses on the internal representation. Therefore the "Open" in OpenAD. The two other frequently mentioned program transformation tools are Adifor (Bischof et al., 1996) and TAMC/TAF (Giering et al., 2005). Not so surprisingly, all these transformation tools have a similar policy regarding the application language: Their primary target is Fortran, and they all more or less accept Fortran95. Except Adifor, they are all working on $\mathrm{C}$ too, although at an experimental stage. Tapenade and OpenAD take advantage of their architecture there, whereas TAF is undergoing a major rewriting to reach "TAC". The $\mathrm{C}++$ target language is a different story, commonly regarded as a hard problem, and postponed for future research.

The ability to run global analyses is a strong point in favor of the program transformation approach. Program transformation AD tools slightly differ from one another 
at the level of the data-flow analyses that they perform. Their internal representation is important here, since it conditions the accuracy of the static data-flow analyses. Also, some tools have specific analyses that allow for slightly improved differentiated code. But in general, the most profitable analyses have spread to all tools. For instance the activity analysis, that finds out whether a given variable somewhere in the code actually has a nontrivial derivative, is available in the four tools above.

In the adjoint mode, the program transformation tools differ in how they address the taping problem ( $c f$ section 2.3 ). There are mostly two approaches, namely "Recompute-All" (RA) for TAMC/TAF and "Store-All" (SA) for Tapenade and OpenAD. There is no adjoint mode in Adifor, but a previous attempt Adjfor, using SA, will serve as a basis for the adjoint mode of OpenAD. Section 3.3 compares the "Recompute-All" and "Store-All" approaches. In reality, it turns out that both approaches must be hybridized, and their performances grow very much alike.

Although it doesn't feature an adjoint mode yet, let's mention the extension of the NAGWare Fortran compiler (Naumann et al., 2005) that includes AD capabilities right inside the NAG Fortran95 compiler. The advantage is that phase 1 of program transformation is done by the compiler, the internal form is already defined, and the final phase 4 is useless. There is a slight difficulty for phases 2 and 3 because separate compilation, which is the standard in compilers, prevents global analyses and transformations. Therefore the differentiated code closely follows the structure of the original code, very much like overloading-based tools do. The adjoint mode is under development, and will follow the code-list approach of overloading tools.

\begin{tabular}{|l||l|l|l|l|}
\hline & target & approach & adjoint & taping strategy \\
\hline \hline OpenAD & F77/F95/(soon)C & transfo. & yes & SA \\
\hline Adifor & F77/F95 & transfo. & no & \\
\hline TAMC/TAF & F77/F95/(soon)C & transfo. & yes & RA \\
\hline Tapenade & F77/F95/(soon)C & transfo. & yes & SA \\
\hline NAG F95 & F77/F95 & compiler & no & \\
\hline Adol-C & C++ & overload. & yes & SA + Code-List \\
\hline ADiMat & MATLAB & overload. & no & \\
\hline MAD & MATLAB & overload. & no & \\
\hline
\end{tabular}

Table 1. Summarized comparison of some AD tools and environments

\subsection{Overloading vs Program Transformation}

Overloading is the ability, available in some languages, to define different implementations for the same procedure or operation. It is the type of the procedure arguments that decides which implementation is chosen for a given call. Object-Oriented programming is a generalization of Overloading. Using overloading, one can redefine the meaning of arithmetical operations to include derivatives computations. AD by 
code overloading requires little modification of the original program: all is needed is to change the declared type of the variables that have a derivative. Still, this must be done generally by hand. Then, after linking to a predefined overloading library for arithmetical operations, the program computes the derivatives.

On the other hand, explicit program transformation requires a very complex $\mathrm{AD}$ tool, similar to a compiler, which parses the original program in order to analyze it and then to create a new program that computes the derivatives. One advantage of this approach is its flexibility: it builds a totally new program, whose structure need not follow the original. The other advantage is the possibility to run a number of global static analyses on the original code, that can lead to interesting optimizations in the differentiated code. The cost of this approach is a long development effort to build the tool, but in theory all that one can do with Overloading can also be done with program transformation.

Specifically for the adjoint mode, overloading has a handicap: it must follow the control flow and execution order of the original program. Since the adjoint mode needs to run the derivative computations in the reverse order, these computations cannot be run by the overloaded operations. The common answer is, instead, to store the required derivative computations themselves on a stack. It is only when the overloaded program terminates that this computation stack is used and actually run in the correct order. In other words, the overloaded statements write a new program, named a "codelist", from its last line to its first line, and only at the end this code-list is executed to get the derivatives. Storing the derivative computations is expensive: the code-list grows with execution time, since it contains no control structure. The values needed in the derivatives computation must still be stored too. A number of refinements can reduce this cost, many of them inspired by the program transformation approach, but overloading is lagging behind. The typical size of applications that can be efficiently adjointed by overloading is notably smaller than by program transformation.

In contrast, the program transformation approach can produce a well structured adjoint algorithm, which is smaller than a code list, and this is done only once at differentiation time. The compiler can exploit this control structure to generate efficient code. Only the values needed in the derivatives computation must be stored.

To conclude this section, one must note that the relative merits of Overloading and Program transformation are getting blurred with time. One drawback of Overloading used to be a noticeable inefficiency of overloaded programs, which is now reduced by very clever compilers. Some overloading tools are now considering to run a preliminary global analysis of the program, and some amount of program transformation to automatically change the types of variables when this is necessary. This amounts to adding activity analysis into the overloading scheme, thus improving the final code. There are also a number of strategies in Adol-C to reduce the size of the code-list. On the other hand, the code-list strategy can be attractive for the program transformation tools, in the cases where reversing the flow of control and the flow of data become too complex. This is not the case at present, but might very well be for AD of Object-Oriented programs. 


\subsection{Storage vs Recomputation}

As mentioned in section 2.3, an adjoint algorithm $\overline{\mathrm{P}}$ must provide a way to retrieve (most of) the intermediate values of $\mathrm{P}$, using a combination of three elementary ways: memory storage, forward recomputation, and backward reversal. In present AD tools, backward reversal is rarely used. Inserting some backward reversal into the general strategy, and finding the best combination, are still open problems. We shall focus here on what is available in real tools, namely storage and recomputation. All strategies radically modify the adjoint algorithm structure of section 2.2.

The TAMC/TAF tool initially relies on recomputation, leading to the RecomputeAll (RA) approach. The RA approach recomputes each needed $W_{k}$ on demand, by restarting the program on input $W_{0}$ until instruction $I_{k}$. The cost is extra execution time, grossly proportional to the square of the number of run-time instructions $p$. Figure 1 summarizes RA graphically. Left-to-right arrows represent execution of original instructions $I_{k}$, right-to-left arrows represent the execution of the differentiated instructions $\overleftarrow{I}_{k}$ which implement $\bar{W}_{k-1}=f_{k}^{\prime t}\left(W_{k-1}\right) \cdot \bar{W}_{k}$. The big black dot represents the storage of all variables needed to restart execution from a given point, which is called a snapshot, and the big white dots represent restoration of these variables from the snapshot.

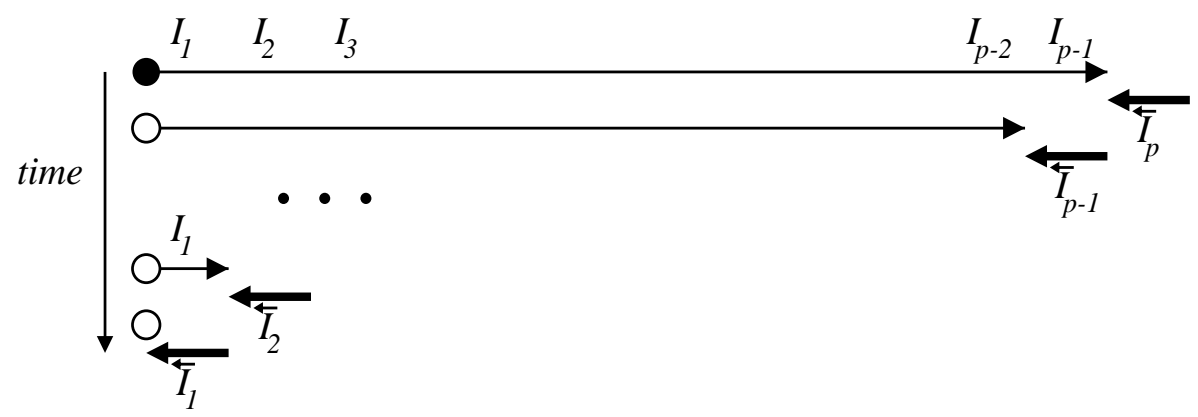

Figure 1. The "Recompute-All" tactic

The Tapenade and OpenAD tools initially rely on storage, leading to the Store-All (SA) approach. The SA approach stores each $W_{k}$ in memory, onto a stack, just before the corresponding $I_{k+1}$ during the forward sweep of $\overline{\mathrm{P}}$. Then during the backward sweep, each $W_{k}$ is restored from the stack before the corresponding $\overleftarrow{I}_{k+1}$. The cost is memory space, essentially proportional to the number of run-time instructions $p$. Figure 2 summarizes SA graphically. Small black dots represent storage of the $W_{k}$ on the stack, before next instruction might overwrite them, and small white dots represent their popping from the stack when needed. We draw these dots smaller than on figure 1 because it turns out we don't need to store all $W_{k}$, but only the variables that will be overwritten by $I_{k+1}$. 


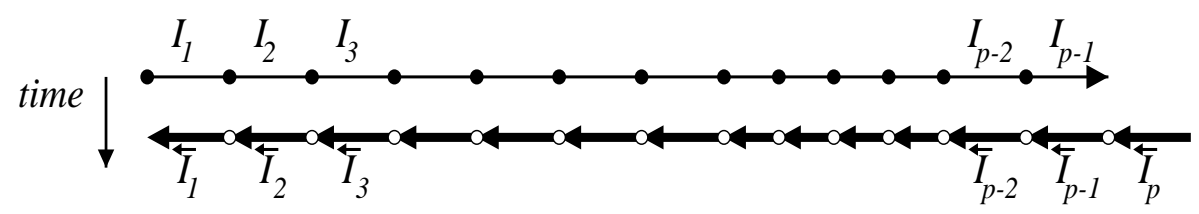

Figure 2. The "Store-All" tactic

The RA and SA approaches appear very different. The quadratic run-time cost of the RA approach appears unacceptable at first sight. However, the TAF tool is successful and performs comparably with Tapenade. One reason for that is the runtime cost of individual storage operations in the SA approach, which must not be overlooked. These operations often damage data locality, thus compromising later compiler optimizations. Values are stored on and retrieved from a dynamic stack, whose management also has some cost. Hardware can provide answers, e.g. prefetching, but these low-level concepts are not easily managed from the target language level.

In any case, pure RA and pure SA are two extreme approaches: the optimum usually lies in-between. Clearly recomputing the result of an expensive program expression can cost far more than simple storage, although costs in memory space and in run time are hard to compare. On the other hand, consider a computation of an indirection index in a loop, such as: nodeIndex $=$ leftEnd (edgeIndex).

Assume that both leftEnd and edgeIndex are available. In addition to its inherent memory space, storage tactic for nodeIndex already costs one push and one pop from the stack, i.e. more than twice the run-time of simple recomputation. Therefore recomputation is here cheaper for both memory space and run time.

But the main reason why RA and SA approaches perform comparably on large simulation codes is that neither of them can work alone anyway! The quadratic run time cost of pure RA is simply unacceptable, and the linear memory space cost of pure SA easily overwhelms the largest disk space available. The classical solution is called checkpointing, and it applies similarly to RA and SA. Checkpointing will be discussed in detail in section 4.1. All we need to say here is that Checkpointing is a storage/recomputation trade-off which can be recursively applied to nested pieces of the program. In ideal situations, optimal checkpointing makes both the run-time increase factor and the memory consumption grow like only the logarithm of the size of the program. In other words if $p$ is the number of run-time instructions of $\mathrm{P}$, then the run-time of $\overline{\mathrm{P}}$ will grow like $p \times \log (p)$ and the maximum memory size used will grow like $\log (p)$. These optimal costs remain the same, whether applied to the RA or SA approaches. This is illustrated on figures 3 and 4: RA and SA approaches visibly come closer as the number of nested checkpointing levels grow. On figure 3, the part on a gray background is a smaller scale reproduction of the basic RA scheme of 
figure 1. Similarly on figure 4, the gray box is a smaller scale reproduction of the basic SA scheme of figure 2. Apart from what happens at these "leaves" (the gray boxes), figures 3 and 4 are identical. The question remains to compare pure SA and pure RA, but it becomes less crucial as these are applied to smaller pieces of the program. We believe SA with some amount of recomputation is more efficient, especially on small pieces of program, because the stack can stay in cache memory. This is why we chose SA as the basis approach for our AD tool Tapenade.

\section{Space-time trade-offs for reversing large simulation codes}

We now focus on the construction of adjoint algorithms for large simulation codes. We recall that the adjoint approach in general is the only practical way to obtain gradients, because of the large number of input parameters and the long simulation run time. The adjoint algorithms obtained through AD belong to this category.

This section deals with the fundamental difficulty of adjoint algorithms namely, the need to retrieve most of the intermediate values of the simulation in reverse order. Section 3.3 described the RA and SA approaches, but neither can work on the present large simulation codes. Both need to be amended through intensive use of checkpointing, which is described in section 4.1. This shows in particular that RA and SA behave similarly when checkpointing comes into play, and we will therefore restrict to the SA approach from then on. Section 4.2 recalls the only known situation where optimal checkpointing can be found. Section 4.3 describes the general situation, where checkpointing is applied on structured programs seen as call trees at the topmost level.

\subsection{Checkpointing}

On large programs P, neither the RA nor the SA approach can work. The SA approach uses too much memory, grossly proportional to the run-time number of instructions. The RA approach consumes computation time, grossly squaring the run-time number of instructions. Both approaches need to use a special trade-off technique, known as checkpointing. The idea is to select one or many pieces of the run-time sequence of instructions, possibly nested. For each piece C, one can spare some repeated recomputation in the RA case, some memory in the SA case, at the cost of remembering a snapshot, i.e. a part of the memory state at the beginning of C. We studied how to keep the snapshot size as low as possible in (Hascoët et al., 2006). The structure of real programs usually forces the pieces to be subroutines, loops, loop bodies, or fragments of straight-line code.

Let us compare checkpointing on RA and SA in the ideal case of a pure straightline program. We claim that checkpointing makes RA and SA come closer. Figure 3 shows how the RA approach can use checkpointing for one program piece $\mathrm{C}$ (the first part of the program), and also for two levels of nested checkpoints. The benefit comes 


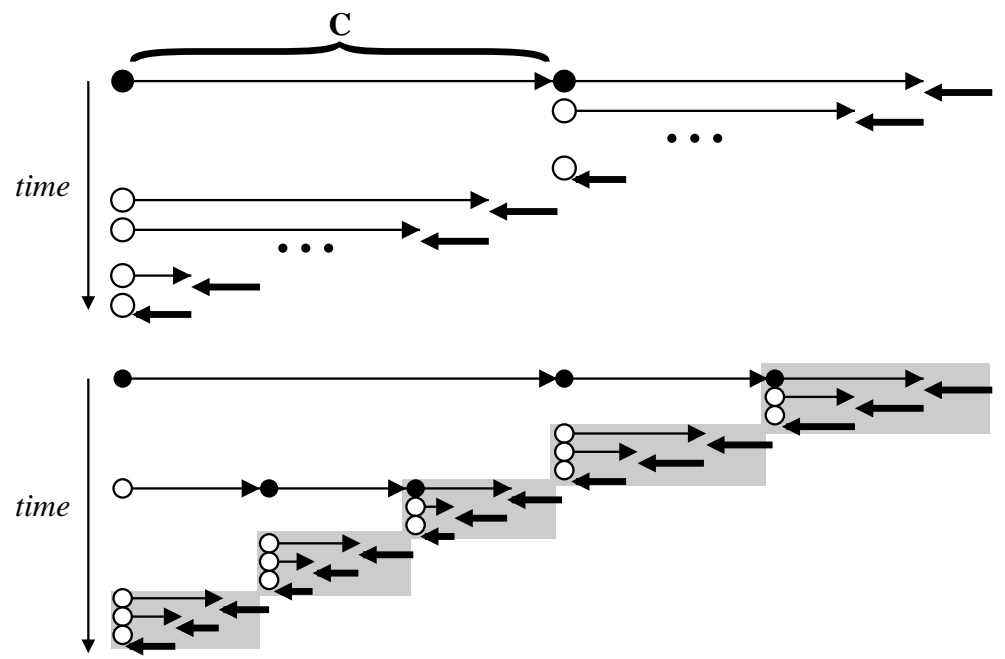

Figure 3. Checkpointing on the "Recompute-All" tactic

from the checkpointed piece being executed fewer times. The cost is memory storage of the snapshot, needed to restart the program just after the checkpointed piece. The benefit is higher when $C$ is at the beginning of the enclosing program piece. On very large programs, 3 or more nested levels can be useful. At the lower levels, the memory space of already used snapshots can be reused. Similarly, figure 4 shows how the SA approach can use the same one-level and two-levels checkpointing schemes. The
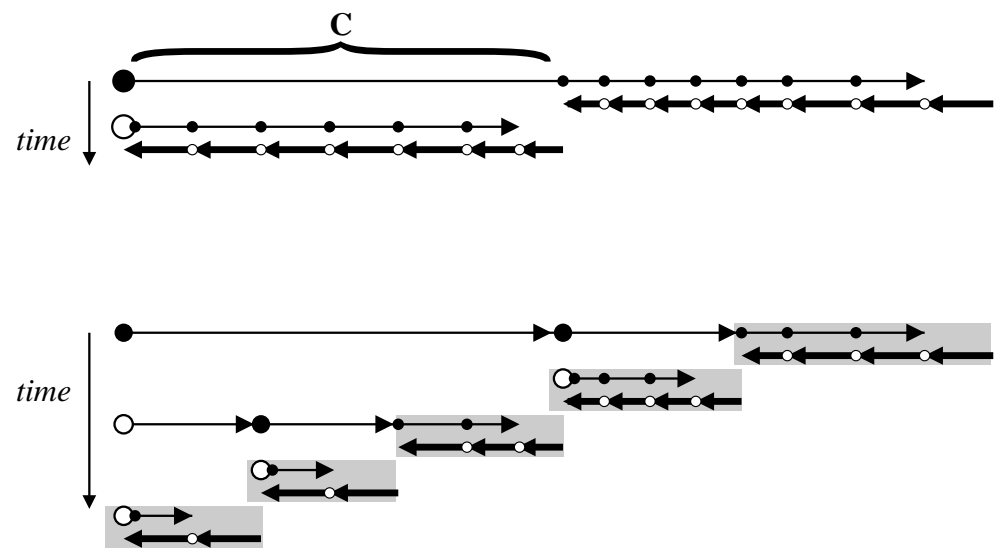

Figure 4. Checkpointing on the "Store-All" tactic

benefit comes from the checkpointed piece being executed the first time without any 
storage of intermediate values. This divides the maximum size of the stack by 2 . The cost is again the memory size of the snapshots, plus this time an extra execution of the program piece C. Again, the snapshot space used for the second level of checkpointing is reused for two different program pieces. Visibly, the two schemes come closer as the number of nested checkpointing levels grow.

Let's analyze more precisely why checkpointing generally improves things. Focusing on the SA approach from the top part of figure 4, the run-time cost of checkpointing is an extra run of $\mathrm{C}$. The benefit lies in the maximum memory size used. Call $\mathrm{D}$ the remaining of the original code, downstream $\mathrm{C}$. With no checkpointing, the maximum memory use is reached at the end of the forward sweep, when all intermediate values computed during $\mathrm{C}$ and then $\mathrm{D}$ are stored. We will write this maximum:

$$
\text { peak }=\operatorname{tape}(C)+\operatorname{tape}(D)
$$

With checkpointing, there are two local maximal memory uses: first at the end of the forward sweep of D,

$$
\text { peak }_{\mathrm{D}}=\operatorname{snp}(\mathrm{C})+\operatorname{tape}(\mathrm{D})
$$

where $\operatorname{snp}(\mathrm{C})$ is the snapshot needed to re-run $\mathrm{C}$, and second at the end of the re-run, i.e. forward sweep, of C,

$$
\operatorname{peak}_{\mathrm{C}}=\operatorname{tape}(\mathrm{C})
$$

To see why peak $_{\mathrm{D}}<$ peak, observe that tape $(\mathrm{C})$ grows linearly with the size of $\mathrm{C}$ : each executed statement overwrites a variable, which must be saved in general. On the other hand $\operatorname{snp}(\mathrm{C})$ is the subset of the memory state at the beginning of $\mathrm{C}$ which is used by $\mathrm{C}$. This set grows slower and slower as $\mathrm{C}$ grows bigger, because the successive assignments during $\mathrm{C}$ progressively hide the initial values. Therefore $\operatorname{snp}(\mathrm{C})$ grows less than linearly with the size of $C$ and for large enough $C$, we have $\operatorname{snp}(C)<\operatorname{tape}(C)$.

\subsection{Checkpointing fixed-length flat code sequences}

Consider the model case of a program P made of a sequence of pieces $I_{k, k=1 \rightarrow p}$, each piece considered atomic and taking the same time. Assume also that each piece consumes the same amount of storage tape $(I)$, that the snapshot $\operatorname{snp}\left(I_{k}\right)$ is sufficient to re-run not only $I_{k}$ but all subsequent pieces, and that all $\operatorname{snp}\left(I_{k}\right)$ have the same size $\operatorname{snp}(I)$. Under those assumptions, it was shown in (Griewank, 1992; Grimm et al., 1996) that there exists an optimal checkpointing scheme, i.e. an optimal choice for the placement of the nested checkpoints.

The optimal checkpointing scheme is defined recursively, for a given maximal memory consumption and a maximal slowdown factor. The memory consumption is expressed as $s$, the maximum number of snapshots that one wishes to fit in memory at the same time. The slowdown factor is expressed as $r$, the maximum number of times one accepts to re-run any $I_{k}$. For a given $s$ and $r, l(s, r)$ is the maximum number of steps that can be adjointed, starting from a stored state which can be a snapshot. It is achieved by running as many initial steps as possible $\left(l_{1}\right)$, then place a snapshot 
and again adjoint as many tail steps as possible $\left(l_{2}\right)$. For the initial sequence, $l_{1}$ is maximized recursively, but this time with only $r-1$ recomputations allowed since one has been consumed already, and for the tail sequence, $l_{2}$ is maximized recursively, but with only $s-1$ snapshots available, since one has been consumed already. Therefore

$$
l(s, r)=l(s, r-1)+l(s-1, r),
$$

which shows that $l(s, r)$ follows a binomial law:

$$
l(s, r)=\frac{(s+r) !}{s ! r !} .
$$

This optimal checkpointing scheme can be read in two ways. First, when the length $p$ of the program grows, there exists a checkpointing scheme for which both the memory consumption and the slowdown factor due to recomputations grow only like $\log (p)$. Second, for real situations where the available memory and therefore the maximal $s$ is fixed, the slowdown factor grows like the $s^{t h}$ root of $p$.

This model case is not so artificial after all. Many simulation codes repeat a basic simulation step a number of times. The steps may vary a little, but still take roughly the same time, tape size, and the snapshot to run one step or $n$ successive steps is basically the same because each step build a state which is the input to the next step. One question remains with the number $p$ of iterations of the basic step. In a few cases $p$ is actually known in advance, but in general it is unknown and the program iterates until some convergence criterion is satisfied. Strictly speaking, this optimal checkpointing scheme can only be organized when $p$ is known in advance. However, there exist approximate schemes with basically the same properties when $p$ is dynamic, i.e. known only at run-time. It is very advisable to apply these schemes, optimal or not, to the principal time-stepping loops of the program to differentiate.

\subsection{Checkpointing call trees}

In many situations the structure of the program $\mathrm{P}$ is not as simple as a sequence of identical steps. Even in the case of iterative simulations, the program structure inside one given step can be arbitrary. Since AD applies to the source of P, we are here concerned with the static structure of $\mathrm{P}$, which is a call graph i.e. a graph of procedures calling one another. Each procedure in turn is structured as nested control constructs such as sequences, conditionals, and loops.

This strongly restricts the possible checkpointing schemes. Generally speaking, the beginning and end of a checkpointing piece $\mathrm{C}$ must be contained in the same procedure and furthermore inside the same node of the tree of control structures. For example a checkpoint cannot start in a loop body and end after the loop. The optimal scheme described in section 4.2 is therefore out of reach. The question is how can one find a good checkpointing scheme that respects these restrictions. 
Let's make the simplifying assumption that checkpointing occurs only on procedure calls. If we really want to consider checkpointing for other program pieces, we can always turn these pieces into subroutines. Figure 5 shows the effect of reverse AD on a simple call graph, in the fundamental case where checkpointing is applied to each procedure call. This is the default strategy in Tapenade. Execution of a procedure A

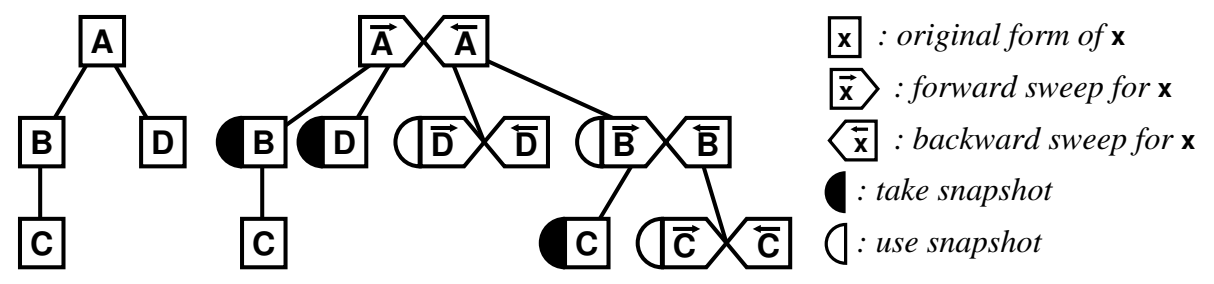

Figure 5. Checkpointing on calls in Tapenade reverse $A D$

in its original form is shown as A. The forward sweep, i.e. execution of A augmented with storage of variables on the stack just before they are overwritten, is shown as $\vec{A}$. The backward sweep, i.e. actual computation of the reverse derivatives of $A$, which pops values from the stack to restore previous values, is shown as $\overleftarrow{A}$. For each procedure call, e.g. B, the procedure is run without storage during the enclosing forward sweep $\vec{A}$. When the backward sweep $\overleftarrow{A}$ reaches B, it runs $\vec{B}$, i.e. B again but this time with storage, and then immediately it runs the backward sweep $\overleftarrow{B}$ and finally the rest of $\overleftarrow{\mathrm{A}}$. Figure 5 also shows the places where snapshots are taken and used to run procedures B, C and D twice. If the program's call graph is actually a well balanced call tree, the memory size as well as the computation time required for the reverse differentiated program grow only like the depth of the original call tree, i.e. like the logarithm of the size of $\mathrm{P}$, which compares well with the model case of section 4.2.

In this framework, the checkpointing scheme question amounts to deciding, for each arrow of the call graph, whether this procedure call must be a checkpointed piece or not. This is obviously a combinatorial problem. With respect to the number of nodes of the call graph, there is no known polynomial algorithm to find the optimal checkpointing scheme. There is no proof that it is NP nor exponential either. In any case, we can devise approximate methods to find good enough schemes.

The fundamental step is to evaluate statically the performance of a given checkpointing scheme. If the call graph is actually a call tree, this can be done using relatively simple formulas. These formulas will use the following data for each node R, i.e. each procedure $\mathrm{R}$ of the call tree:

- time $(R)$ : the average run-time of the original procedure $R$, not counting called subroutines.

$-\overline{\operatorname{time}}(\mathrm{R})$ : the average run-time of the forward and backward sweeps of the adjoint algorithm of $R$, not counting called subroutines. 
- tape $(\mathrm{R}, i)$ : the memory size used to store intermediate values of $\mathrm{R}$ on the tape, between its $i^{\text {th }}$ subroutine call and the following procedure call. Indices $i$ start at 1 up, and imax stands for the last $i$.

$-\operatorname{snp}(\mathrm{T}, i)$ : the memory size used to store the snapshot to checkpoint the $i^{\text {th }}$ subroutine called by the root procedure of $\mathrm{T}$.

- snp_time $(\mathrm{T}, i)$ : the time needed to write and read the snapshot, very roughly proportional to $\operatorname{snp}(\mathrm{T}, i)$.

These values can be either measured through run-time profiling of the adjoint code, guessed through profiling on the original program, or even grossly evaluated statically on the source program at differentiation time. The formulas also use the navigation primitives:

$-\operatorname{root}(\mathrm{T})$, the procedure at the root of the sub-call-tree $\mathrm{T}$,

$-\operatorname{child}_{i}(\mathrm{~T})$, the $i^{\text {th }}$ sub-call-tree of $\mathrm{T}$,

as well as the boolean $\operatorname{ckp}(\mathrm{T}, i)$, true when the considered scheme does checkpoint the $i^{\text {th }}$ subroutine call made by the root procedure of T. This boolean function indeed defines one checkpointing scheme, and for each "ckp" we define the following durations:

- time $(\mathrm{T})$ as the total run-time cost of $\mathrm{T}$,

- $\overline{\text { time }}(\mathrm{T})$ as the total run-time cost of $\overline{\mathrm{T}}$, the adjoint algorithm of $\mathrm{T}$.

We can compute these durations, recursively on each sub-tree $\mathrm{T}$ of the call tree, as follows:

$$
\begin{aligned}
& \operatorname{time}(\mathrm{T})=\operatorname{time}(\operatorname{root}(\mathrm{T}))+\sum_{i} \operatorname{time}\left(\operatorname{child}_{i}(\mathrm{~T})\right) \\
& \overline{\operatorname{time}}(\mathrm{T})=\overline{\operatorname{time}}(\operatorname{root}(\mathrm{T}))+ \\
& \sum_{i} \overline{\operatorname{time}}\left(\operatorname{child}_{i}(\mathrm{~T})\right)+\left\{\begin{array}{c}
\text { if } \operatorname{ckp}(\mathrm{T}, i): \operatorname{time}\left(\operatorname{child}_{i}(\mathrm{~T})\right)+\operatorname{snp} \operatorname{time}(\mathrm{T}, i) \\
\text { otherwise }: 0
\end{array}\right.
\end{aligned}
$$

Figure 6 shows an example call tree, together with an example checkpointing scheme, namely on the calls to B, E, and F. The resulting adjoint algorithm is shown on the right. Inspection of the adjoint call tree justifies the above timing evaluations. Similarly, we can evaluate the peak memory consumption during the adjoint algorithm. Arrows on figure 6 show the places where this peak consumption may occur. The formulas are slightly more complex and we need to introduce the intermediate value $\overline{\text { part_mem }}(\mathrm{T})$ in order to compute the final result peak_mem $(\mathrm{T})$. We define

- $\overline{\text { part_mem }}(\mathrm{T}, i)$ as the amount of memory used between the beginning of the forward sweep $\overrightarrow{\mathrm{T}}$ and the $(i+1)^{t h}$ subroutine call done by $\overrightarrow{\mathrm{T}}$. In particular, the last $\overline{\text { part_mem }}(\mathrm{T}$, imax $)$ is the amount of memory used by the complete $\overrightarrow{\mathrm{T}}$. We define $\overline{\text { part_mem }}(\mathrm{T})=\overline{\text { part_mem}}(\mathrm{T}$, imax $)$. 

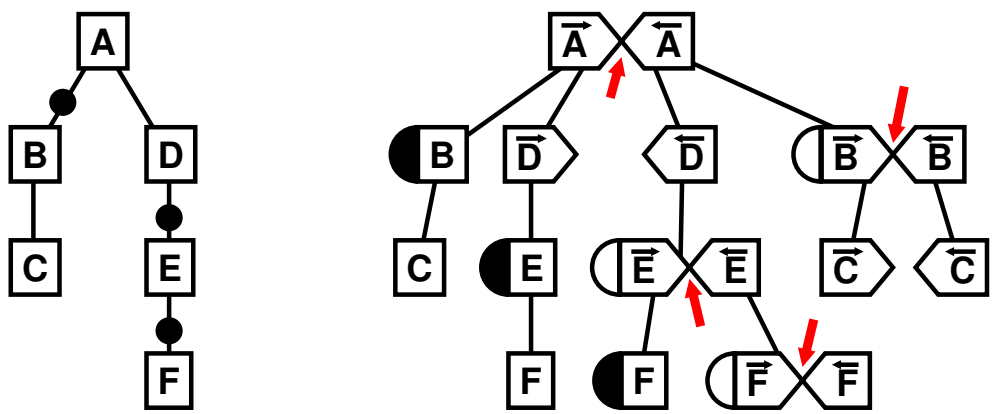

Figure 6. Example Checkpointing scheme on a Call Tree $\overline{\mathrm{T}}$.

- $\overline{\text { peak_mem }}(\mathrm{T})$ as the peak memory consumption during execution of the whole

We can compute these memory consumptions recursively on each sub-tree $\mathrm{T}$ as follows. Notice that $\overline{\text { part_mem}}(\mathrm{T}, i)$ on a given $\mathrm{T}$ is computed recursively for $i$ increasing from 0 to imax, i.e. from the beginning of $\vec{T}$, incorporating progressively each successive call made by $\overrightarrow{\mathrm{T}}$.

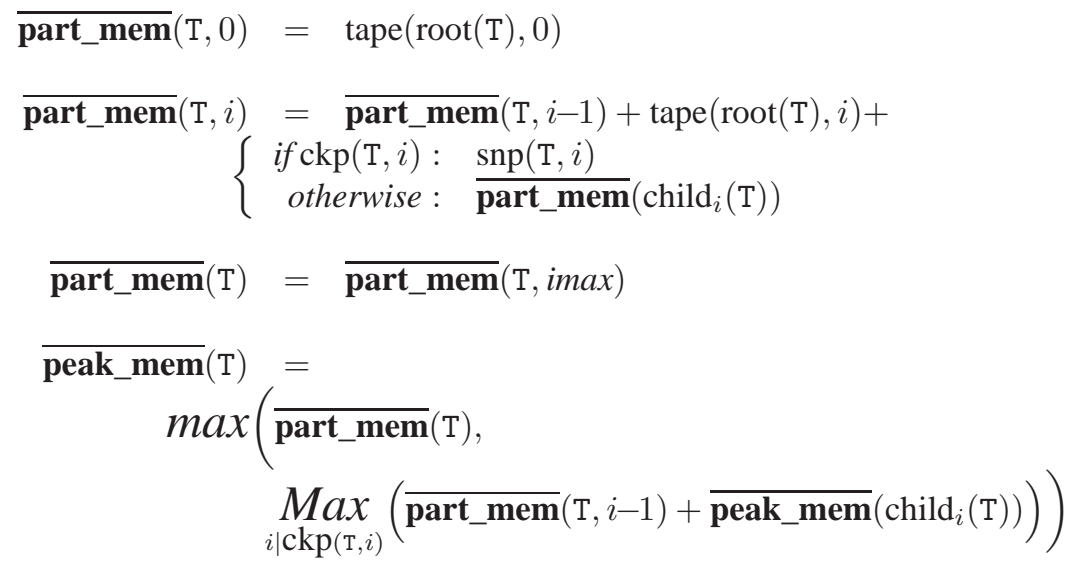

These formulas can be used to evaluate the time and memory consumption for every checkpointing scheme, and therefore to search for an optimal scheme. A simple heuristic could be to sweep through the call tree to find the procedure call (the $i^{t h}$ in T) that gives the best improvement when toggling $\operatorname{ckp}(\mathrm{T}, i)$, actually toggle it, and repeat the process until no improvement remains. We are currently experimenting such a system inside Tapenade. The next section presents some preliminary results. 


\section{Applications}

Prior to experiments, we developed an extension to the default checkpointing strategy of Tapenade: Each subroutine call can now be checkpointed or not, through a boolean directive called C $\$ A D$ NOCHECKPOINT, inserted by hand by the end-used into the original code. In the future, Tapenade will look for a good checkpointing scheme following the principles of section 4.3. Then the end-user can refine this choice through directives. Until this exists, we place these directives by hand on every procedure call to make experiments. We present the results on three large instationnary simulation codes.

\subsection{Stics}

Stics is a Fortran 77 code that simulates the growth of a field, taking into account the plants that are actually grown, the type of soil, the dates and quantities of the inputs in water and nitrates, and the weather during a complete agricultural cycle. This simulation is of course instationnary, with an explicit time-stepping scheme. Stics is developed by the French Institut National pour la Recherche Agronomique (INRA) since 1996.

For this experiment, the goal was to compute the gradient of the total amount of biomass produced, with respect to most of the input parameters. This is a typical application for $\mathrm{AD}$ adjoint mode. For the particular application the simulation ran for about 400 days, i.e. 400 time steps.

The original simulation code is about 27000 lines long, and one simulation runs for 0.4 seconds on a $3 \mathrm{GHz} \mathrm{PC}$.

For this size of program, checkpointing is of course compulsory for the adjoint algorithm. Even with Tapenade's default strategy, i.e. checkpointing on all calls, the peak memory size was larger than the available 2 Gigabytes of memory.

The immediate answer was to apply a slightly sub-optimal version of the checkpointing strategy described in section 4.2 to the toplevel loop of 400 time steps. As a result, the adjoint algorithm actually worked, and returned a gradient that we could validate.

However the slowdown factor from the original simulation to the adjoint algorithm was much too high. We claimed in section 2 that this factor was typically 7 , but on Stics we observed a factor closer to 100 !

In addition to the "optimal" checkpointing strategy applied to the toplevel loop, we looked for a good checkpointing scheme inside the call tree of each time step. We measured the time and memory data from section 4.3 and we found 6 subroutines where it was obviously counter-productive to apply checkpointing. Actually these subroutines behaved very strangely, since for them the snapshot was much larger than 
the tape, which is very unusual. The snapshots were so large that the times to take and read the snapshots, "snp_time" dominated the actual derivative computations.

Using the new predicate in Tapenade, we implemented this checkpointing scheme by explicitly not checkpointing the 6 procedure calls. As a result, we observed a reduction both in time and in peak memory. The slowdown is now only 7.4, and the peak memory is only 80 Megabytes, comparable to the 30 Megabytes static memory size of the original simulation code.

This application shows that a good checkpointing scheme is vital for AD adjoint algorithms of long simulations. In addition, although checkpointing is generally a trade-off that spares peak memory at the cost of run-time slowdown, there are extreme cases where checkpointing looses in both respects and should be avoided.

\subsection{Gyre}

Gyre is a simple configuration of the oceanography code OPA 9.0. It simulates the behavior of a rectangular basin of sea water put on the tropics between latitudes $15^{\circ}$ and $30^{\circ}$, with the wind blowing to the East. OPA 9.0 is developed by the LOCEANIPSL team in Paris VI university. It consists of about 110000 lines of Fortran 90. The Gyre simulation runs for 4320 time steps ranging on 20 days.

The time advancing uses an implicit scheme with a preconditioned conjugate gradient for solving the linear system at each time step. One simulation of the Gyre configuration takes 92 seconds on a $3 \mathrm{GHz} \mathrm{PC}$.

In this experiment, in order to perform data assimilation, the goal was to obtain a gradient of an objective function, the total heat flux across some boundary at the northern angle, with respect to an initial parameter, which is the temperature field in the complete domain 20 days before. This is a typical application for the adjoint mode of $\mathrm{AD}$.

Here again, the checkpointing strategy for iterative loops of section 4.2 is absolutely necessary. Otherwise, the adjoint code exceeded 3 Gigabytes of memory after just 80 time steps. With the checkpointing strategy on iterative loops, the adjoint code computed an exact gradient with the following performances:

- time: 748 seconds, i.e. a slowdown of 8.2

- memory: 494 Megabytes, to be compared with the 40 Megabytes static memory size of the original code.

The next improvement step is to look for a better checkpointing scheme inside the time steps: we progressively increased the number of procedure calls that are not checkpointed, until we used all the available memory of 3 Gigabytes. As expected, this reduced the number of duplicate executions due to checkpoints, and thus reduced the slowdown factor to 5.9. We believe this can still be improved with some work. 
Incidentally, notice that for the previous version 8.0 of OPA, the adjoint code was written by hand, which is a long development. The slowdown factor was between 3 and 4, which is a little better than what we obtain with Tapenade. However, the hand adjoint reduced the memory consumption by storing states only at certain time steps, and recomputing them during the backward sweep by interpolation. This proves efficient, but still this is a cause for inaccuracy in the gradient. In comparison, the AD adjoint algorithm returns a more accurate gradient for a relatively small extra cost.

Figure 7 shows a part of the resulting gradient, with respect to the initial temperature distribution at a depth of 300 meters. We also show the interpretation of some of its structures made by the oceanographers.

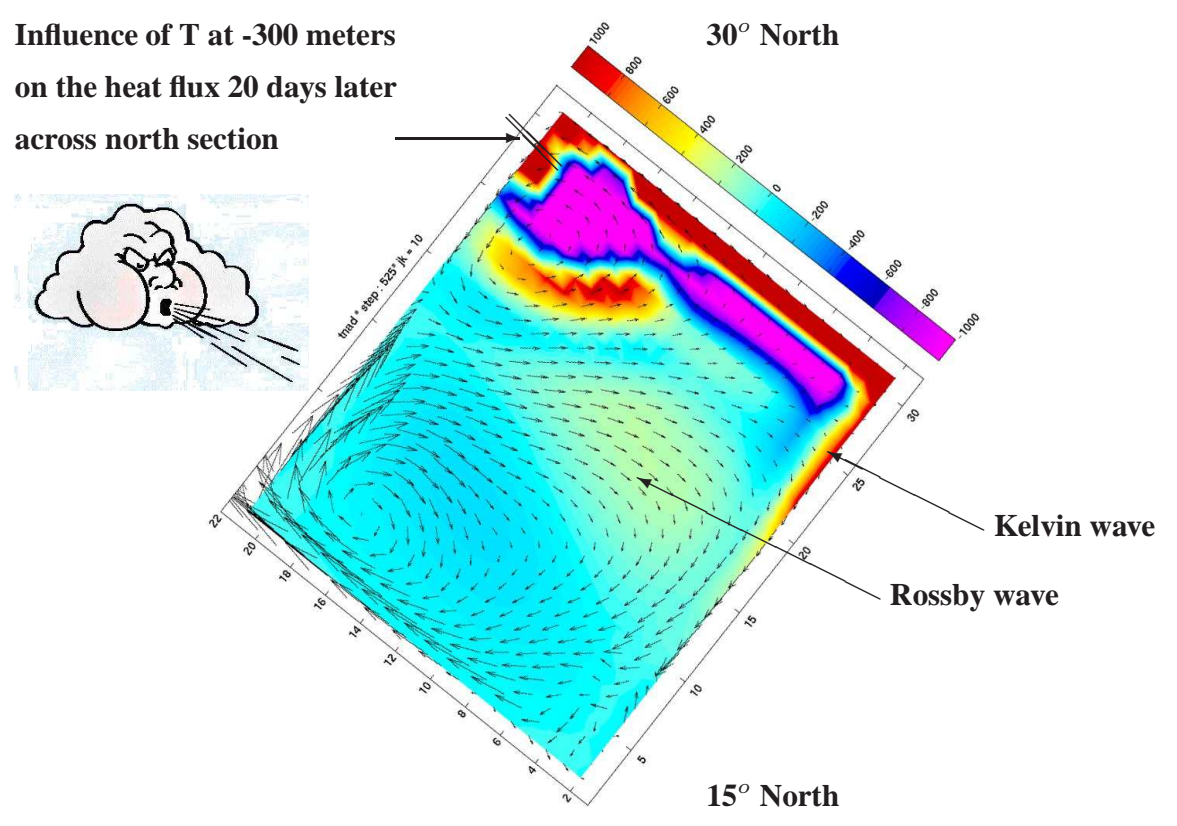

Figure 7. Oceanography gradient by reverse AD on OPA 9.0/Gyre

\subsection{Nemo}

Nemo is a much larger configuration of OPA 9.0, encompassing the North Atlantic. Again, we want to build a gradient through adjoint AD. Work is ongoing and we have few results. We recently obtained our first valid gradient. When this gradient is fully reliable for the whole simulation (450 time steps here) we also expect to have at hand the first version of the checkpointing profiling heuristic described in sec- 
tion 4.3. We plan to use Nemo as our first large testbed to improve this checkpointing profiling heuristic.

\section{Outlook}

We presented the current research on Automatic Differentiation, aiming at automatic generation of efficient adjoint codes for long instationnary simulations. We described the principles of $\mathrm{AD}$, which show that the adjoint mode is certainly the most reasonable way to obtain the code for the gradient of a simulation. We suggested how existing AD approaches and tools could compare in this respect. However, adjoint codes require complex data flow and memory traffic. AD tools have yet to reach the level where these questions are properly solved. In this paper, we tried to present the directions we are considering to address these questions.

The issue of finding good checkpointing schemes on large arbitrary programs is central. There is a strong suspicion that this combinatorial question is NP-hard. We believe we can devise good heuristics, suggesting efficient checkpointing schemes even for large applications. Yet, these heuristics rely on static data-flow analyses that are always approximate, and also on approximate models of the performance of a code on a computer architecture. Therefore interaction with the end-user is definitely necessary to obtain really efficient checkpointing schemes.

Although our example applications are still at an experimental level, we hope they show that $\mathrm{AD}$ tools are making constant progress, and produce adjoint codes whose efficiency is similar to hand-coded adjoints. In a matter of years, we will probably be able to relieve numericians from the tedious task of hand-writing adjoints.

\section{References}

Bischof C., Carle A., Khademi P., Mauer A., " ADIFOR 2.0: Automatic Differentiation of Fortran 77 Programs", IEEE Computational Science \& Engineering, vol. 3, n 3, p. 18-32, 1996.

Bischof C., Lang B., Vehreschild A., “Automatic Differentiation for MATLAB Programs”, Proceedings in Applied Mathematics and Mechanics, vol. 2, $\mathrm{n}^{\circ}$ 1, p. 50-53, 2003.

Bücker M., Corliss G., Hovland P., Naumann U., Norris B. (eds), Automatic Differentiation: Applications, Theory, and Implementations, vol. 50 of Lecture Notes in Computer Science and Engineering, Springer, 2006.

Corliss G., Faure C., Griewank A., Hascoët L., Naumann U. (eds), Automatic Differentiation of Algorithms, from Simulation to Optimization, LNCSE, Springer, 2001.

Forth S., " An Efficient Overloaded Implementation of Forward Mode Automatic Differentiation in MATLAB", ACM Transactions on Mathematical Software, vol. 32, n² 2, p. 195-222, 2006. 
Giering R., Kaminski T., Slawig T., " Generating Efficient Derivative Code with TAF: Adjoint and Tangent Linear Euler Flow Around an Airfoil”, Future Generation Computer Systems, vol. 21, n 8, p. 1345-1355, 2005. http://www.FastOpt.com/papers/Giering2005GED.pdf.

Griewank A., " Achieving logarithmic growth of temporal and spatial complexity in reverse automatic differentiation", Optimization Methods and Software, vol. 1, n 1, p. 35-54, 1992.

Griewank A., Evaluating Derivatives: Principles and Techniques of Algorithmic Differentiation, Frontiers in Applied Mathematics, SIAM, 2000.

Griewank A., Juedes D., Utke J., " Adol-C: a package for the automatic differentiation of algorithms written in C/C++”, ACM Transactions on Mathematical Software, vol. 22, p. 131167, 1996. http://www.math.tu-dresden.de/ adol-c.

Grimm J., Pottier L., Rostaing N., " Optimal Time and Minimum Space-Time Product for Reversing a Certain Class of Programs”, in M. Berz, C. H. Bischof, G. F. Corliss, A. Griewank (eds), Computational Differentiation: Techniques, Applications, and Tools, SIAM, p. 95106, 1996.

Hascoët L., Dauvergne B., " The Data-Flow Equations of Checkpointing in Reverse Automatic Differentiation”, in Alexandrov et al (ed.), Computational Science - ICCS 2006, vol. 3994 of Lecture Notes in Computer Science, Springer, p. 566-573, 2006.

Hascoët L., Pascual V., TAPENADE 2.1 User's Guide, Technical Report n 300, INRIA, 2004. http://www.inria.fr/rrt/rt-0300.html.

Mani K., Mavriplis D.-J., “ An Unsteady Discrete Adjoint Formulation for Two-Dimensional Flow Problems with Deforming Meshes”, AIAA Aerospace Sciences Meeting, Reno, NV, AIAA Paper 2007-0060, 2007.

Naumann U., Riehme J., “A Differentiation-Enabled Fortran 95 Compiler”, ACM Transactions on Mathematical Software, 2005.

Utke J., Naumann U., OpenAD/F: User manual, Technical report, Argonne National Laboratory, 2006. http://www.mcs.anl.gov/openad/.

Verma A., " ADMAT: Automatic Differentiation in MATLAB Using Object Oriented Methods”, in M. E. Henderson, C. R. Anderson, S. L. Lyons (eds), Object Oriented Methods for Interoperable Scientific and Engineering Computing: Proceedings of the 1998 SIAM Workshop, SIAM, Philadelphia, p. 174-183, 1999. 